\title{
Multitaper Time-frequency Peak-based Longitudinal Sleep Analysis in 5XFAD Mouse Model
}

\section{Chaeyeon Kim}

Gwangju Institute of Science and Technology

\section{Victor James Drew}

Gwangju Institute of Science and Technology

\section{Mincheol Park}

Gwangju Institute of Science and Technology

\section{Tae Kim ( $\nabla$ tae-kim@gist.ac.kr)}

Gwangju Institute of Science and Technology https://orcid.org/0000-0003-0201-5401

\section{Research}

Keywords: Multitaper spectrogram, TF-peak detection, sleep spindles, 5XFAD, Alzheimer's disease (AD)

Posted Date: August 6th, 2021

DOI: https://doi.org/10.21203/rs.3.rs-779142/v1

License: (c) (1) This work is licensed under a Creative Commons Attribution 4.0 International License. Read Full License 


\title{
Multitaper time-frequency peak-based longitudinal sleep analysis in 5XFAD mouse model
}

\author{
Chaeyeon Kim, Victor James Drew*, Mincheol Park, Tae Kim*
}

Department of Biomedical Science and Engineering, Gwangju Institute of Science and Technology, Gwangju 61005, Republic of Korea

\begin{abstract}
Background: Sleep disturbance is common in Alzheimer's disease (AD), but the characteristics of sleep disturbance remain unclear. Multitaper spectral analysis (MSA) is a novel method for investigating sleep. However, MSA-based sleep research in AD is lacking; hence we applied MSA to examine the sleep of AD.
\end{abstract}

Methods: Electroencephalograms were recorded on 3-, 6-, and 10-month-old 5XFAD mice, and the time-frequency (TF) peaks were detected using MSA. We comparatively analyzed the TF peaks between genotypes and age groups.

Results: The sigma TF peaks ( $\sim 80 \%)$ were sleep spindles. MSA-based TF plot showed distinct patterns, agreeing with manual scoring. With $\mathrm{AD}$ progression, the characteristics of TF peaks coherently changed; shorter sigma TF peaks outnumbered longer ones; dark-period fast spindle TF peak density decreased significantly at both 6 and 10 months.

Conclusions: Multitaper TF peaks might provide biomarkers for the progression of AD. Further investigations are warranted.

Keywords: Multitaper spectrogram, TF-peak detection, sleep spindles, 5XFAD, Alzheimer's disease $(\mathrm{AD})$

\footnotetext{
${ }^{\#}$ These authors equally contributed to this manuscript

${ }^{*}$ Corresponding author.

E-mail address: tae-kim@ gist.ac.kr (T. Kim)
} 


\section{Introduction}

Alzheimer's disease (AD) is the most common form of dementia, consisting of memory loss and behavioral changes, and is characterized by amyloid-beta plaque accumulation and neurofibrillary tangle aggregation [1]. Sleep disruption is increasingly recognized as a symptom of AD with severe implications on quality of life. Sleep disturbances occur in up to $44 \%$ of patients with AD [2]. In the early stages of $\mathrm{AD}$, patients report diminished sleep in the form of sleep fragmentation and nighttime awakenings, and other changes in sleep characteristics, including daytime drowsiness. Currently, there is no cure for $\mathrm{AD}$, but treatment during the early stages of $\mathrm{AD}$ may delay or prevent further progression [3], providing a strong incentive for early diagnosis. Among the existing techniques for diagnosing AD through biomarkers, electroencephalography (EEG) may offer a non-invasive and cost-effective method for early detection and progressive monitoring of $\mathrm{AD}[4]$.

EEG is an electrophysiological monitoring method to record the electrical activity of the brain. The intervals of the spontaneous electrical activity of the brain, also known as brainwaves, are recorded using the multiple non-invasive electrodes placed along the scalp [5]. Standard EEG analysis involves the identification of patterns such as the amplitude, frequency, and shape of brainwaves. EEG provides records of brain activity, enabling a unique approach towards the diagnosis of neuropathic diseases and sleep disorders $[6,7]$.

The spontaneous neural oscillations observed in EEG recordings consist of multi-frequency and non-stationary signals from localized brain areas. The current standard for detecting neural oscillations utilizes the time-domain detection method, which traces oscillations based on a threshold of high amplitude $[8,9]$. However, the time-domain detection method is less effective at distinguishing noises from the target signal and dividing multi-frequency signals, which can detect false negatives. This suggested the need for detection in the time-frequency domain, strengthening the advantages and compensating for the disadvantages of both time and frequency domains.

The time-frequency analysis calculates the spectral estimation of EEG oscillatory dynamics based on the Fourier transform. In practice, applying the Fourier transform on finite data results in a 
tradeoff between the time and frequency resolution according to Heisenberg's uncertainty principle [10]. MSA is a novel method for investigating sleep, which provides the optimized tradeoff between bias and variance. MSA is capable of detecting the dynamics of sleep EEG at a wide range of time scales [11]. However, MSA-based sleep research in AD is currently lacking. In this study, we applied the TF-peak analysis method of MSA on 5XFAD mice to determine whether it detects changes in pathologic oscillations from longitudinal sleep in a mouse model of AD. Further analysis looked into the morphological characteristics of TF-peaks, such as log-prominence and duration, that can be used to distinguish the 5XFAD phenotype and potentially serve as a parameter that can precisely predict disease progression.

\section{Methods}

\section{Animal model}

The 5XFAD transgenic mice (Stock No. 34840, Jackson Laboratory) were used as an animal model of AD. 5XFAD mice overexpress amyloid precursor protein (APP) with Swedish (K670N, M671L), Florida (I716V), and London (V717I) mutations, as well as human PS1 with M146L and L286V mutations under the control of the murine Thy-1-promotor. These mice are of B6SJLF1/J background and have high APP expression correlating with accelerated production and accumulation of amyloid-beta 42 (Aß42). Mice were separated based on age and genotype: Control groups (3-month: $\mathrm{n}=12 ; 6$-month: $\mathrm{n}=11 ; 10$-month: $\mathrm{n}=12$ ) and 5 XFAD groups (3-month: $\mathrm{n}=12 ; 6$-month: $\mathrm{n}=11 ; 10$-month: $\mathrm{n}=10$ ). All mice were handled according to GIST LARC guidelines for animal care. Animals were housed in a temperature-controlled room at $23{ }^{\circ} \mathrm{C} \pm 2{ }^{\circ} \mathrm{C}$ with a $12 / 12 \mathrm{~h} \mathrm{light/dark}$ cycle (light on at 7 A.M.). Food and water were provided ad libitum.

\section{EEG implantation surgery}

At 3,6, and 10-months, mice underwent EEG implantation surgery, in which an intracranial 
EEG head mount and intramuscular EMG electrodes were implanted into each mouse, followed by 1-2 weeks of recovery time. The mice were anesthetized before the surgery with $4 \%$ isoflurane and maintained with 0.5 to $1.5 \%$ isoflurane in a stereotaxic frame, and the ketofen $(0.1 \mathrm{mg} / \mathrm{kg})$ was injected subcutaneously to control pain. The EEG electrodes were injected into the five holes on the exposed cranium, with $0.8 \mathrm{~mm}$ diameter at reference (AP: $-5.3 \mathrm{~mm}$, ML: $0.0 \mathrm{~mm}$ ), parietal (AP: $-3.5 \mathrm{~mm}$, ML: $1.0 \mathrm{~mm}$ ), frontal (AP: $1.0 \mathrm{~mm}$, ML: $1.0 \mathrm{~mm}$ ), anchor (AP: $-2.0 \mathrm{~mm}, \mathrm{ML}:-1.5 \mathrm{~mm}$ ), and ground (AP: $2.0 \mathrm{~mm}$, ML: $1.5 \mathrm{~mm}$ ). EMG electrodes were inserted into the neck muscles. The wire and the connector (Pinnacle Technology Inc., Oregon, US) were fixed on the highest point of the skull, connected by dental cement and soldering. After recovery from surgery, EEG and EMG signals were recorded for 24 consecutive hours.

The signals were recorded and analyzed using Sirenia software (Pinnacle PAL 8200) with 2 kHz sampling and a $100 \mathrm{~Hz}$ low-pass filter. Visual scoring was conducted manually using Sirenia Sleep software (Pinnacle Technology Inc., Oregon, US), followed by the analysis of the sleep structure and EEG/EMG power spectrum. The recording session was divided into 10 -second epochs of continuous data. The vigilance state of each epoch was designated as wake, NREM sleep, and REM sleep according to the EEG and EMG data patterns along with a synchronized video. Wakefulness was characterized by low amplitude and mixed frequencies in the rhythmic waveform of EEG, accompanied by increased EMG activity. NREM sleep signals display high amplitude, irregular EEG waves with a delta power peak, along with low EMG amplitude. REM sleep EEG was characterized by low amplitude, saw-tooth shaped waves (regular waveform) with peak theta power around 7Hz, and flat EMG signals.

\section{Sleep spindle detection algorithm}

The automatic sleep spindle detection followed a custom MATLAB algorithm used in Uygun et al.[12], based on clinical spindle analysis from Ferrarelli et al.[13] The sigma frequency range was band-filtered at $10-15 \mathrm{~Hz}$ for NREM epochs scored manually based on vision. The filter has been designed with a MATLAB designfilt function, the Butterworth filter, consisting of a stop-band 
frequency of 3 and $22 \mathrm{~Hz}$, and the pass-band frequency of 10 and $15 \mathrm{~Hz}$. The filtered EEG data was transformed using root mean square (RMS) and cubed for noise reduction and signal recognition. Sleep spindles were determined with two thresholds: a lower threshold of $1.2 \mathrm{x}$ mean cubed RMS for duration $>0.5 \mathrm{~s}$ and $<10 \mathrm{~s}$, and an upper threshold of $3.5 \mathrm{x}$ cubed RMS. The detailed adjustment of the interspindle interval condition of 0.01 to $0.2 \mathrm{~s}$ was then applied. Sleep spindle density was calculated by dividing the sleep spindle count by the total NREM sleep duration (min.).

\section{TF-peak detection algorithm}

TF-peaks were detected using an algorithm developed by Dimitrov et al., designed to identify transient oscillations in the time-frequency domain [14]. EEG input of time-domain data was transformed into the time-frequency domain with the multitaper spectrogram. The multitaper spectrogram was calculated using the Chronux mtspecgramc function in MATLAB. The multitaper was designed using the 9 DPSS tapers having a window length $(\mathrm{N})$ of $2.5 \mathrm{~s}$ with a step length of $0.05 \mathrm{~s}$ and frequency resolution $(\Delta \mathrm{f})$ of $4 \mathrm{~Hz}$ and time-half-bandwidth $(\mathrm{TW})=5$. The frequency range limitation was 0 to $60 \mathrm{~Hz}$ at the multitaper spectrogram. In each frequency band (delta, theta, sigma, beta, and gamma), the TF-peaks were detected selectively within the bandwidth range of the prominence values: $0.5-4 \mathrm{~Hz}$ for delta, $4-7 \mathrm{~Hz}$ for theta, $10-15 \mathrm{~Hz}$ for sigma, $15-30 \mathrm{~Hz}$ for beta, and $30-50 \mathrm{~Hz}$ for gamma band, respectively. The prominence value from the computed multitaper spectrogram detected the local maximum in both frequency and time domains with the findpeaks function in MATLAB.

TF-peaks were identified at two separate axes with a fixed order. First, the peaks are found at a frequency domain - the maximum prominence (max-prominence) peak is selected between the given range of the bandwidth at the spectra at each time point. Next, the algorithm detects the peaks at the time domain among the serial sequence of the max-prominence peaks from the spectra, making the peak distinguishable as being the local maximum at both the time and frequency domain.

Noise peaks were selected with the k-means clustering (unsupervised method). Data were 
clustered into two classes, and the cluster with the higher prominence was selected to be the candidate peak group. The candidates were filtered to have the central frequency value of the given ranges. Also, peak distances less than $0.3 \mathrm{~s}$, and frequency bandwidths less than half of the spectral resolution from the multitaper spectrogram were eliminated.

The detected TF-peaks were measure for morphological properties (log prominence, duration, bandwidth, etc.). The prominence value represented the power of the TF-peak - the height of the peak relative to the nearby peaks. Using the prominence instead of the absolute value of the height enables the referencing of the local power level at the spectrum. Prominence values were transformed into a logarithmic scale reflecting the distribution of the detected prominence. The time-domain characteristic of the detected TF-peaks was represented as a duration. The duration was calculated to have a halfprominence width in the time axis of the spectrogram, which represented the time interval - the start point to the endpoint of the TF-peak. On the other hand, the frequency domain characteristic consisted of three parameters: central frequency, minimum frequency, and maximum frequency. As the duration represented the time domain characteristic of the local maximum point of the TF-peak, the central frequency showed the characteristic at the frequency domain. Thus, the central frequency represents the frequency value of the max-prominence peak from the spectrum. The minimum and maximum frequency provided the lowest/highest frequency value from TF-peak. Count and density parameters represent the numeric value of the TF-peak. The count refers to the total number of TF-peaks during the total recording time, and the density is calculated with the count value divided by the total sleep time according to the corresponding sleep state.

\section{Statistical analysis}

The statistical significance of the data was conducted using R software. The Shapiro-Wilk test was utilized for the testing normality of each data group. Depending on the normality test results, ANOVA or Kruskal-Wallis tests were performed, followed by post hoc tests. 


\section{Results}

\section{Sleep spindles account for the majority of sigma TF-peaks in 5XFAD mice}

The sleep spindle detection algorithm and TF-peak detection algorithm were applied to the sleep-recording data. Sleep spindles from the time-domain overlapped with the sigma TF-peaks on the multitaper spectrogram (Fig. 1A). Sleep spindles consisted of transient oscillations of the sigma frequency range captured by sigma TF-peaks. The agreement between the sleep spindles and sigma TFpeaks were counted in both longitudinal control and 5XFAD mouse groups (Fig. 1B). The control mice showed an agreement rate of over $81 \%$, higher than the agreement rate reported in human patients from three independent datasets (mean agreement: $53 \pm 4 \%$ ) [14]. A high agreement rate was also observed in 5XFAD mice, but it declined with age.

\section{Multitaper spectrogram reflects vigilance status and longitudinal pathology in mice}

The full 24-hour recordings from each mouse were analyzed in the multitaper spectrogram, which was matched with the hypnogram at its corresponding time trace. The patterns of the multitaper spectrogram were matched with the sleep states from the hypnogram in control mice (Fig. 2A). Wakefulness showed strong theta power along with blob-like regions in various frequency ranges under 10Hz. In NREM sleep, delta power was the most dominant frequency band, and it contained numerous theta and sigma peaks. Meanwhile, REM sleep showed a consistent broadband frequency range of strong alpha and theta power and a relatively weaker delta frequency range (Fig. 2B).

The multitaper spectrogram with the hypnogram in longitudinal data of 5XFAD mice is displayed in Figure 3. Changes of multitaper spectrogram with aging revealed the sleep disruption in 5XFAD mice. Wakefulness was increased in both light and dark periods, but especially in dark periods (Fig. 3B and 3C). The slowing of EEG in vigilance states of 5XFAD mice was apparent when comparing the longest duration of wakefulness in 6-month 5XFAD mice (Fig. 3B) with the longest duration of 
wakefulness in 10-month 5XFAD mice (Fig. 3C).

\section{Refining the definition of TF-peak enables more precise detection of sleep spindles}

We computed the morphological characteristics such as log-prominence, central frequency, and duration for the detected sleep spindles and sigma TF-peaks. The bandwidth was also calculated only for the sigma TF-peaks. The histograms of longitudinal sigma TF-peak morphological characteristics showed a right-skewed distribution in log-prominence and central frequency. The histogram for the duration and bandwidth showed double peaks (Fig. 4). Because sleep spindles are defined as having a duration over $0.5 \mathrm{~s}$, the TF-peak detection algorithm was refined to eliminate the sigma TF peaks shorter than $0.5 \mathrm{~s}$. Comparing the distributions of morphological characteristics from the refined TF-peaks with the sleep spindles, we found the refined TF-peaks showed more similarities in the distribution of central frequency and log prominence than the original definition of TF-peaks. The morphological characteristics of sleep spindles and TF-peaks were also similar in different age groups after the refinement. Interestingly, between the double peaks of the duration histogram, the longer-duration peak was always taller than the shorter-duration peak in WT. However, in 5XFAD, the shorter-duration peak became taller progressively through six and ten months of age.

\section{TF-peak parameters in 5XFAD mice at various frequency bands progressively changed with aging}

TF-peak parameters of each vigilance state were further analyzed with bandwise TF-peak detection. In the waking state, the delta and theta TF-peaks increased in 5XFAD mice (Fig. 5). More specifically, in 5XFAD mice, wake delta TF-peak prominence and frequency, and theta TF-peak count also increased over time (Fig. 5A and B). Wake TF-peak counts increased longitudinally in 5XFAD mice for both beta and gamma frequencies. In contrast, only wake beta TF-peaks in 5XFAD showed a higher frequency range than those of age-matched control mice (Fig. 5C and 5D). 
A decline in delta and sigma activity was apparent among the NREM TF-peak morphological characteristics (Fig. 5E and 5F). NREM delta and sigma TF-peak counts decreased in 6 and 10-monthold 5XFAD mice (Fig. 5E). In 5XFAD mice, the sigma TF-peak showed a decline in duration and central frequency value (Fig. 5F).

The notable longitudinal changes of REM TF-peak morphological characteristics appeared as REM EEG slowing in 5XFAD mice (Fig. 5G and 5H). REM delta TF-peak prominence increased, and REM theta TF-peak minimum and maximum frequencies declined over time. REM delta TF-peak prominence significantly increased in 5XFAD mice, and the REM theta TF-peak from 5XFAD mice showed elevated duration compared to control mice at 10-months.

\section{TF-peak parameters for sleep spindles sensitively detected disease progression}

To explore the diagnostic parameters for the progression of AD pathology, we further analyzed the TF-peaks at the entire frequency range of the sleep spindles $(9-17 \mathrm{~Hz})$. The TF-peaks parameters showed significant declines in duration, frequency, and count in 5XFAD at ten months, while WT remained unchanged except duration. The count of TF peaks showed the most significant reduction $(\%$, $\mathrm{p}<0.0001$; Fig. 6A). Additionally, we performed subgroup analysis to find conditions to detect the changes at an earlier stage of six months. All of the detected TF-peaks were classified into subgroups based on the recording timing (light vs. dark) and central frequency (fast vs. slow spindle; $12-17 \mathrm{~Hz}$ vs. $9-12 \mathrm{~Hz}$, respectively) $[14,15]$. We found that TF-peak count and density of the fast spindles decreased most prominently during the dark period (Fig. $6 \mathrm{~B}$ and C).

\section{Discussion}

Here we demonstrated that MSA could detect a large proportion of sleep spindles with sigma TF peaks. Also, TF-peak parameters changed significantly with the disease progression in the AD mouse model. Interestingly, the sigma TF peaks with short duration $(<0.5 \mathrm{~s})$ outnumbered that of longer 
duration at 10 months in 5XFAD mice. Fast spindle TF peak counts during the dark period showed significant decreases at both six and ten months. To the best of our knowledge, this study is the first report for the application of MSA on sleep analysis of AD and shows compelling evidence to support the superior ability to detect disease progression using sleep analysis.

\section{Sigma TF-peaks as a marker of sleep spindles}

The agreement between sleep spindles detected by the conventional algorithm (REF. McNally paper) and sigma TF-peaks in our study was higher than in human application. Interestingly, control mice maintained a similar level of agreement in all three age groups, but 5XFAD mice showed a decline with increased age. In patients with Alzheimer's disease and mild cognitive disorder the fast sleep spindle density decrease [16]. Also, spindle density during NREM stage 2 sleep was suggested as an early biomarker for neuronal dysfunction [17]. Consistent with previous reports, our findings indicate that brain activities detected as the sigma TF peaks but mismatched with standard sleep spindle criteria increases with the progression of Alzheimer's disease.

\section{Bandwise TF-peak parameters as biomarkers of disease progression}

Regarding morphological characteristics of sigma TF-peaks, the duration of the sleep spindle showed a broader range of values than that of TF-peaks. The duration distribution difference may be due to multiple TF-peaks included in single sleep spindle durations. On the other hand, sigma TF-peaks detected as having duration under $0.5 \mathrm{~s}$ do not follow the classical definition of sleep spindles, implying that sigma band oscillations are not necessarily sleep spindles. Therefore, the sigma TF-peaks may represent a broader form of neural oscillation beyond sleep spindles. These concepts are supported by reports of reduced sleep spindle amplitude and duration in amnestic mild cognitive impairment (aMCI) and $\mathrm{AD}$ patients, as well as by the use of sleep spindle duration as a biomarker of $\mathrm{AD}$ [18].

We found that 5XFAD mice showed progressive changes in TF-peak parameters of different 
frequency bands and vigilance states, similar to changes seen in EEG power-based sleep analyses in patients with $\mathrm{AD}$. The increased delta and theta EEG power with decreased alpha and beta activities is frequently seen in patients with $\mathrm{AD}$ [19-21]. In our data, an increase in wake delta TF-peak prominence was significant at six months, possibly indicating drowsiness or abnormal wakefulness. The EEG slowing in $\mathrm{AD}$ is most prominent in wake and REM states, and REM EEG slowing is correlated to cognitive impairment [22]. The frequency ranges of TF-peaks for wake delta, REM delta, and REM theta increased in 5XFAD mice, supporting the literature. Reportedly, abnormal delta and theta band activities in $\mathrm{AD}$ can be restored by long-term acetylcholinesterase inhibitor therapy [23]. Hence, increased wake and REM delta TF-peak prominence may reflect the cholinergic deficits in AD, which relates to the cognitive deficits in $\mathrm{AD}$ patients.[24] However, the frequency range of wake beta TF peaks increased in 5XFAD, which may appear to contradict the overall trend. This finding may be due to the altered sensitivity to noradrenergic blockade in $\mathrm{AD}$, as shown in TgCRND8 mice [25, 26]. 5XFAD mice have also been reported to have elevated noradrenaline levels in 5-month-old male mice. REM theta TF-peaks in 5XFAD mice showed a progressive increase in TF-peak duration, which can be interpreted as a homeostatic response to the decreased REM theta TF-peak count [27].

\section{TF-peak parameters in NREM sleep}

In addition to the abnormalities observed in wake and REM states, the NREM state showed abnormal EEG activities, including reduced delta and sigma activity in TF-peaks in 5XFAD mice over time. The decline in NREM delta TF-peak count and density indicates that the ability to generate the NREM delta is progressively disrupted in AD. The slow wave activity (SWA) reduction is associated with a high concentration of amyloid beta 42 in cerebrospinal fluid (CSF) and impaired sleep-dependent consolidation of declarative memory. These relationships support the hypothesis of disrupted slow wave generation by A $\beta$ pathology $[28,29]$. In APP transgenic mice, SWA is reduced, especially in the slow oscillation frequency range $(<1 \mathrm{~Hz})$ by three months of age, which is two months before $\mathrm{A} \beta$ plaques are apparent. Reduced GABA levels and downregulated $\mathrm{GABA}_{\mathrm{A}}$ and $\mathrm{GABA}_{\mathrm{B}}$ receptor expression directly 
impacted the generation of slow oscillations by disrupting neuronal synchrony [30]. Because NREM delta TF-peak count decreased in 5XFAD mice, the unresolved sleep pressure may be accumulated. As a result, daytime sleepiness increased, which were reflected in increased wake theta TF-peak count and density. Wake theta and NREM delta TF-peak counts were negatively correlated (r: $-0.83, p<0.01)$ in 10-month-old 5XFAD mice [31].

\section{Fast spindle TF peak during the dark period as a sensitive biomarker}

The decreased NREM sigma TF-peak duration and counts in 5XFAD mice can signify that sleep spindles decreased. Spindle density is negatively correlated with CSF A $\beta 42$ a biomarker of AD $[17,22]$. The NREM sigma TF-peak showed a decline in the central frequency, implying that the sleep spindle frequency was slowed down as described in $\mathrm{AD}$ patients. The alteration of sleep spindles in $\mathrm{AD}$ and MCI correlated only to fast sleep spindles [16]. Indeed, our subgroup analysis revealed that the density of TF peaks for fast sleep spindle $(12-17 \mathrm{~Hz})[14,15]$ decreased progressively at six and ten months of age. Taken together, we suggest that TF peak for fast sleep spindles can be a sensitive biomarker of disease progression in 5XFAD mice.

\section{Conclusion}

We confirmed that MSA could characterize sleep states and identifies longitudinal pathological changes in 5XFAD mice. TF peaks for sleep spindles could reliably predict the sleep spindles and serve as a more sensitive marker for the longitudinal progression of $\mathrm{AD}$. Moreover, the refinement by eliminating sigma TF-peaks with a duration shorter than $0.5 \mathrm{~s}$ enabled the detection of sleep spindles by TF peaks comparable to the conventional spindle detection algorithm in both genotypes. Furthermore, we suggest that the fast-sigma band TF-peak during the dark period might serve as a sensitive biomarker for $\mathrm{AD}$ progression. 


\section{Figure legends}

Figure 1. Sigma TF-peaks and sleep spindles in mice. (A) Multitaper spectral analysis of EEG. Gray shade indicates the sleep spindles detected by the conventional algorithm, and rectangles with black dotted lines denote sigma TF-peaks in 6-month control mice. (B) The agreement between sleep spindle and sigma TF-peak was tabulated.

Figure 2. The multitaper spectrogram reflects the vigilance status of control mice. (A) A representative multitaper spectrogram and a hypnogram of 24-hour EEG recording of a 6-month-old control mouse. In the hypnogram (top), pink shades indicate wakefulness, purple NREM sleep, and REM sleep green. The spectrogram (middle and bottom) is designed with the 9 DPSS tapers having window length $(\mathrm{N})$ of $2.5 \mathrm{~s}$ with the step of $0.05 \mathrm{~s}$ and frequency resolution $(\Delta \mathrm{f})$ of $4 \mathrm{~Hz}$ and bandwidth $(\mathrm{TW})=5$, and power is described in $\mathrm{dB}$ with provided color scale bars. (bottom) An enlarged oneminute segment of the spectrogram from zeitgeber time 8 to 9. The detected TF-peaks were labeled with the black dotted line on the spectrogram. Rectangles with black dotted lines denote sigma TFpeaks. (B) Representative multitaper spectrograms of 200 second period spent in wakefulness (pink), NREM sleep (purple), and REM sleep (green).

Figure 3. The longitudinal changes of EEG in 5XFAD mice by the multitaper spectrogram. (A) A representative multitaper spectrogram and a hypnogram of 24-hour EEG recording of a 3-month-old control mouse. In the hypnogram (top), pink shades indicate wakefulness, purple NREM sleep, and REM sleep green. The spectrogram (middle and bottom) is designed with the 9 DPSS tapers having window length $(\mathrm{N})$ of $2.5 \mathrm{~s}$ with the step of $0.05 \mathrm{~s}$ and frequency resolution $(\Delta \mathrm{f})$ of $4 \mathrm{~Hz}$ and bandwidth $(\mathrm{TW})=5$, and power is described in $\mathrm{dB}$ with provided color scale bars. (bottom) An enlarged oneminute segment of the spectrogram from zeitgeber time 8 to 9. (B and C) Figures with same formats of a representative 6- and 10-month-old 5XFAD mouse, respectively.

Figure 4. Morphological parameters of original and revised sigma TF peaks and sleep spindles. (A-C) Control mice. (D-F) 5XFAD mice. (A and D; B and E; C and F) Age groups of 3, 6, 10 months, respectively. (A-F) Histograms of morphological parameters for the sigma TF peaks, 
including log prominence (upper left), central frequency (upper right), duration (lower left) for each panel. Red lines indicate the histogram of the original sigma TF peak, blue sleep spindle, and green revised TF peak (duration $>0.5 \mathrm{~s}$ ).

Figure 5. Morphological parameters of the bandwise TF peaks during wakefulness, NREM and REM sleep in control and 5XFAD mice. (A-D) Genotype and age group comparisons of morphological parameters, count, state time, and density for delta, theta, beta, and gamma frequency bands. Parameters were normalized to the value of 3-month-old control mice. (E and F) Same comparisons of delta and sigma TF peaks during NREM sleep. (G and H) Same comparisons of delta and theta TF peaks during NREM sleep. Blue horizontal lines indicate the comparison between genotypes; black horizontal lines between age groups. ${ }^{*} \mathrm{p}<0.05 ; * * \mathrm{p}<0.01 ; * * \mathrm{p}<0.001 ; * * * \mathrm{p}<$ 0.0001 by ANOVA or Kruskal-Wallis test followed by post hoc pairwise comparisons.

Figure 6. Parameters of TF peaks for sleep spindles and subgroup analysis. (A) Morphological parameters of sleep spindle frequency range $(9-17 \mathrm{~Hz})$. Parameters were normalized to the value of 3month-old control mice. (B and C) The count and the density of TF peaks for fast (9-12Hz) and slow $(12-17 \mathrm{~Hz})$ spindle frequency during light and dark periods. Green dots and lines indicate 5XFAD mice and blue dots and lines control mice. $\quad * \mathrm{p}<0.05 ; * * \mathrm{p}<0.01 ; * * * \mathrm{p}<0.001 ; * * * \mathrm{p}<0.0001$ by ANOVA or Kruskal-Wallis test followed by post hoc pairwise comparisons.

\section{Abbreviations}

AD: Alzheimer's disease; MSA: Multitaper spectral analysis; TF: time-frequency; EEG: electroencephalography; APP: amyloid precursor protein; A $\beta 42$ : amyloid-beta 42; RMS: root mean square; WT: wild type; ANOVA: repeated measure analysis of variance; aMCI: amnestic mild cognitive impairment; SWA: slow wave activity; CSF: cerebrospinal fluid; GABA: gamma-aminobutyric acid; REM: rapid eye movement; NREM: non-REM 


\section{Declarations}

\section{Authors' contributions}

Chaeyeon Kim: Investigation, Methodology, Data analysis, Writing-original draft. Victor James Drew: Conceptualization, data collection, Writing-original draft. Mincheol Park: Methodology, S $\mathrm{t}$ analysis. Tae Kim: Conceptualization, Supervision, Fund acquisition, Writing-review \& editing.

\section{Funding}

This work was supported by National Research Foundation of Korea (NRF) grant funded by the Korean government (Ministry of Science and ICT, 2017R1A5A1014708 and 2018 R1A2B6002804 to T.K.; Ministry of Education, 2015R1D1A1A01059119 to T.K.); 2021 Joint Research Project of Institutes of Science and Technology to T.K.

\section{Availability of data and material}

The datasets used and/or analyzed in the current study are available from the corresponding authors on reasonable request.

\section{Acknowledgements}

Not applicable

\section{Ethics approval and consent to participate}

All work involving animals approved by the ethics committee at Gwangju Institute of Science and Technology (GIST-2020-031) which is fulfilled with Association for Assessment and Accreditation of Laboratory Animal Care International guidelines. 


\section{Consent for publication}

All the authors have approved the manuscript.

\section{Competing interests}

The authors declare no competing interests.

\section{References}

1. Uddin MS, Stachowiak A, Mamun AA, Tzvetkov NT, Takeda S, Atanasov AG, Bergantin LB, Abdel-Daim MM, Stankiewicz AM: Autophagy and Alzheimer's Disease: From Molecular Mechanisms to Therapeutic Implications. Front Aging Neurosci 2018, 10:04.

2. Carpenter BD, Strauss M, Patterson MB: Sleep disturbances in community-dwelling patients with Alzheimer's disease. Clinical Gerontologist 1996, 16:35-49.

3. Scheltens P, Blennow K, Breteler MM, de Strooper B, Frisoni GB, Salloway S, Van der Flier WM: Alzheimer's disease. Lancet 2016, 388:505-517.

4. Sarazin M, de Souza LC, Lehericy S, Dubois B: Clinical and research diagnostic criteria for Alzheimer's disease. Neuroimaging Clin NAm 2012, 22:23-32,viii.

5. Niedermeyer E, Lopes da Silva FH: Electroencephalography : basic principles, clinical applications, and related fields. 5th edn. Philadelphia: Lippincott Williams \& Wilkins; 2005.

6. Siddiqui MM, Srivastava G, Saeed SH: Diagnosis of insomnia sleep disorder using short time frequency analysis of PSD approach applied on EEG signal using channel ROC-LOC. Sleep Sci 2016, 9:186-191.

7. Smith SJ: EEG in the diagnosis, classification, and management of patients with epilepsy. $J$ Neurol Neurosurg Psychiatry 2005, 76 Suppl 2:ii2-7. 
8. Sharmila A, Geethanjali P: Effect of filtering with time domain features for the detection of epileptic seizure from EEG signals. J Med Eng Technol 2018, 42:217-227.

9. Jacobs J, Levan P, Chatillon CE, Olivier A, Dubeau F, Gotman J: High frequency oscillations in intracranial EEGs mark epileptogenicity rather than lesion type. Brain 2009, 132:1022-1037.

10. Babadi B, Brown EN: A review of multitaper spectral analysis. IEEE Trans Biomed Eng 2014, 61:15551564.

11. Prerau MJ, Brown RE, Bianchi MT, Ellenbogen JM, Purdon PL: Sleep Neurophysiological Dynamics Through the Lens of Multitaper Spectral Analysis. Physiology (Bethesda) 2017, 32:60-92.

12. Uygun DS, Katsuki F, Bolortuya Y, Aguilar DD, McKenna JT, Thankachan S, McCarley RW, Basheer R, Brown RE, Strecker RE, McNally JM: Validation of an automated sleep spindle detection method for mouse electroencephalography. Sleep 2019, 42.

13. Ferrarelli F, Huber R, Peterson MJ, Massimini M, Murphy M, Riedner BA, Watson A, Bria P, Tononi G: Reduced sleep spindle activity in schizophrenia patients. Am J Psychiatry 2007, 164:483-492.

14. Dimitrov T, He M, Stickgold R, Prerau MJ: Sleep spindles comprise a subset of a broader class of electroencephalogram events. Sleep 2021.

15. Molle M, Bergmann TO, Marshall L, Born J: Fast and slow spindles during the sleep slow oscillation: disparate coalescence and engagement in memory processing. Sleep 2011, 34:1411-1421.

16. Gorgoni M, Lauri G, Truglia I, Cordone S, Sarasso S, Scarpelli S, Mangiaruga A, D'Atri A, Tempesta D, Ferrara M, et al: Parietal Fast Sleep Spindle Density Decrease in Alzheimer's Disease and Amnesic Mild Cognitive Impairment. Neural Plast 2016, 2016:8376108.

17. Kam K, Parekh A, Sharma RA, Andrade A, Lewin M, Castillo B, Bubu OM, Chua NJ, Miller MD, Mullins AE, et al: Sleep oscillation-specific associations with Alzheimer's disease CSF biomarkers: novel roles for sleep spindles and tau. Mol Neurodegener 2019, 14:10.

18. Liu S, Pan J, Tang K, Lei Q, He L, Meng Y, Cai X, Li Z: Sleep spindles, K-complexes, limb movements and sleep stage proportions may be biomarkers for amnestic mild cognitive impairment and Alzheimer's disease. 
Sleep Breath 2020, 24:637-651.

19. Brenner RP, Ulrich RF, Spiker DG, Sclabassi RJ, Reynolds CF, 3rd, Marin RS, Boller F: Computerized EEG spectral analysis in elderly normal, demented and depressed subjects. Electroencephalogr Clin Neurophysiol 1986, 64:483-492.

20. Coben LA, Danziger WL, Berg L: Frequency analysis of the resting awake EEG in mild senile dementia of Alzheimer type. Electroencephalogr Clin Neurophysiol 1983, 55:372-380.

21. Giaquinto S, Nolfe G: The EEG in the normal elderly: a contribution to the interpretation of aging and dementia. Electroencephalogr Clin Neurophysiol 1986, 63:540-546.

22. D'Atri A, Scarpelli S, Gorgoni M, Truglia I, Lauri G, Cordone S, Ferrara M, Marra C, Rossini PM, De Gennaro L: EEG alterations during wake and sleep in mild cognitive impairment and Alzheimer's disease. iScience 2021, 24:102386.

23. Jelic V, Dierks T, Amberla K, Almkvist O, Winblad B, Nordberg A: Longitudinal changes in quantitative EEG during long-term tacrine treatment of patients with Alzheimer's disease. Neurosci Lett 1998, 254:85-88.

24. Mizuno S, Kameda A, Inagaki T, Horiguchi J: Effects of donepezil on Alzheimer's disease: the relationship between cognitive function and rapid eye movement sleep. Psychiatry Clin Neurosci 2004, 58:660-665.

25. Kalinin S, Polak PE, Lin SX, Sakharkar AJ, Pandey SC, Feinstein DL: The noradrenaline precursor LDOPS reduces pathology in a mouse model of Alzheimer's disease. Neurobiol Aging 2012, 33:1651-1663.

26. Colby-Milley J, Cavanagh C, Jego S, Breitner JC, Quirion R, Adamantidis A: Sleep-Wake Cycle Dysfunction in the TgCRND8 Mouse Model of Alzheimer's Disease: From Early to Advanced Pathological Stages. PLoS One 2015, 10:e0130177.

27. Park SH, Weber F: Neural and Homeostatic Regulation of REM Sleep. Front Psychol 2020, 11:1662.

28. Mander BA, Marks SM, Vogel JW, Rao V, Lu B, Saletin JM, Ancoli-Israel S, Jagust WJ, Walker MP: betaamyloid disrupts human NREM slow waves and related hippocampus-dependent memory consolidation. Nat Neurosci 2015, 18:1051-1057.

29. Varga AW, Wohlleber ME, Gimenez S, Romero S, Alonso JF, Ducca EL, Kam K, Lewis C, Tanzi EB, 
Tweardy S, et al: Reduced Slow-Wave Sleep Is Associated with High Cerebrospinal Fluid Abeta42 Levels in Cognitively Normal Elderly. Sleep 2016, 39:2041-2048.

30. Kastanenka KV, Hou SS, Shakerdge N, Logan R, Feng D, Wegmann S, Chopra V, Hawkes JM, Chen X, Bacskai BJ: Optogenetic Restoration of Disrupted Slow Oscillations Halts Amyloid Deposition and Restores Calcium Homeostasis in an Animal Model of Alzheimer's Disease. PLoS One 2017, 12:e0170275.

31. Vyazovskiy VV, Tobler I: Regional differences in NREM sleep slow-wave activity in mice with congenital callosal dysgenesis. J Sleep Res 2005, 14:299-304. 


\section{Figures}
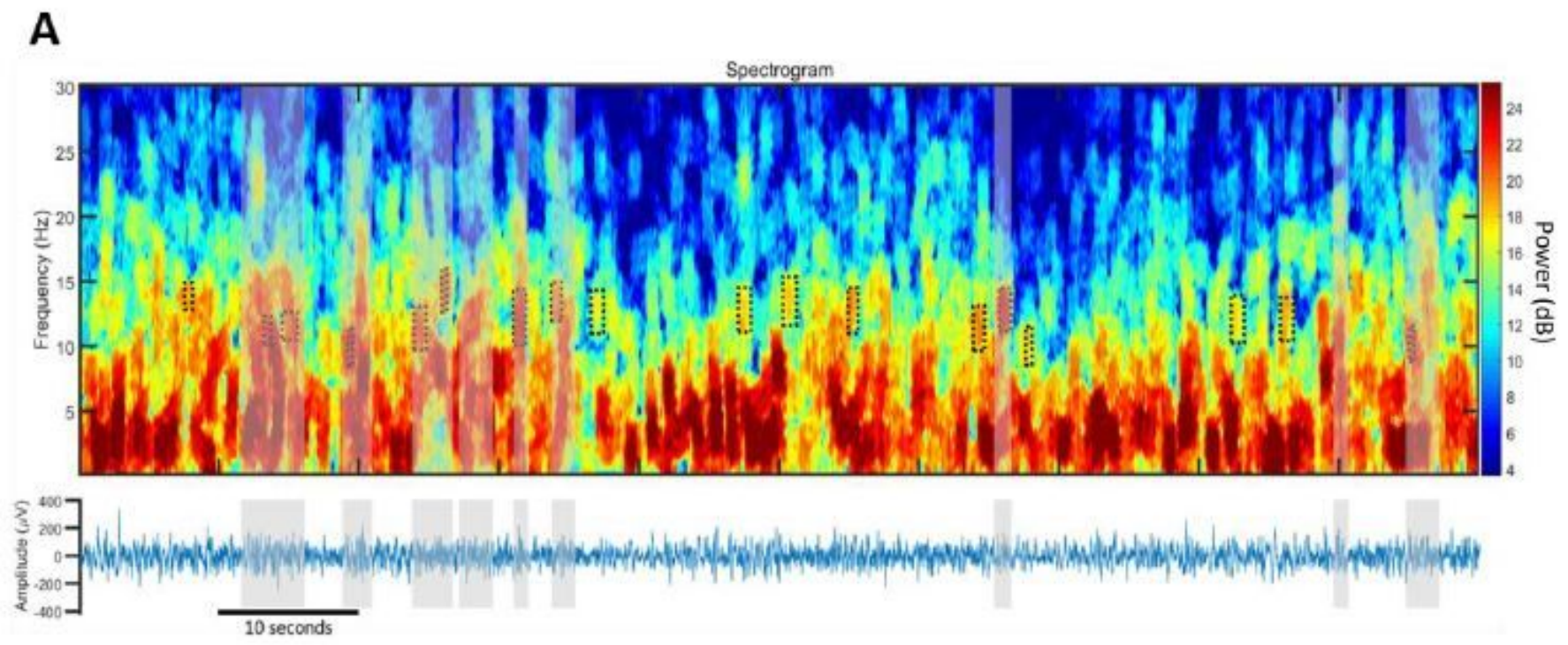

B

\begin{tabular}{|c|c|c|}
\hline & \multicolumn{2}{|c|}{ Agreement } \\
\hline Age & Control & 5XFAD \\
\hline 3-month & $81.65 \%$ & $82.89 \%$ \\
6-month & $81.37 \%$ & $80.49 \%$ \\
10-month & $81.96 \%$ & $73.49 \%$ \\
\hline
\end{tabular}

\section{Figure 1}

Sigma TF-peaks and sleep spindles in mice. (A) Multitaper spectral analysis of EEG. Gray shade indicates the sleep spindles detected by the conventional algorithm, and rectangles with black dotted lines denote sigma TF-peaks in 6-month control mice. (B) The agreement between sleep spindle and sigma TF-peak was tabulated. 
A
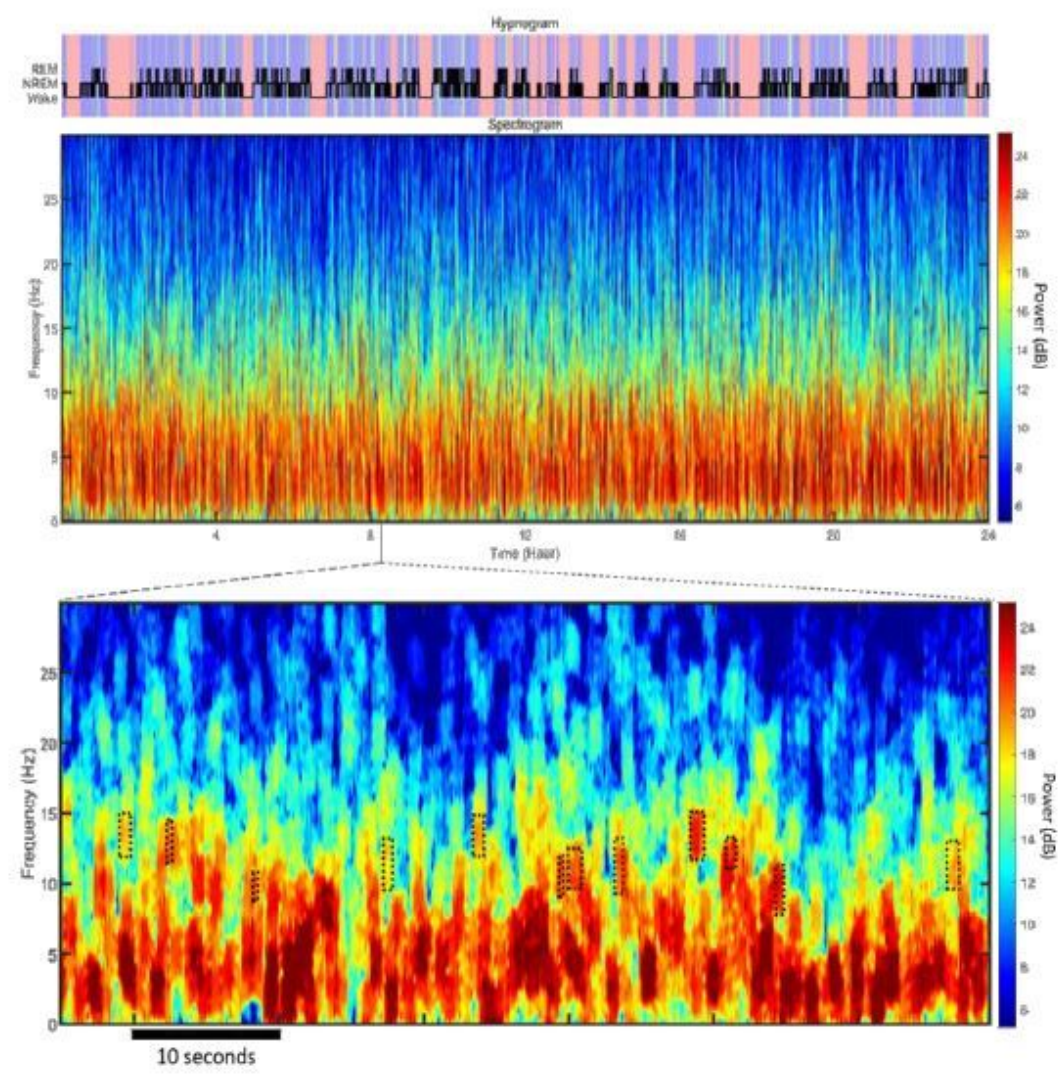

B

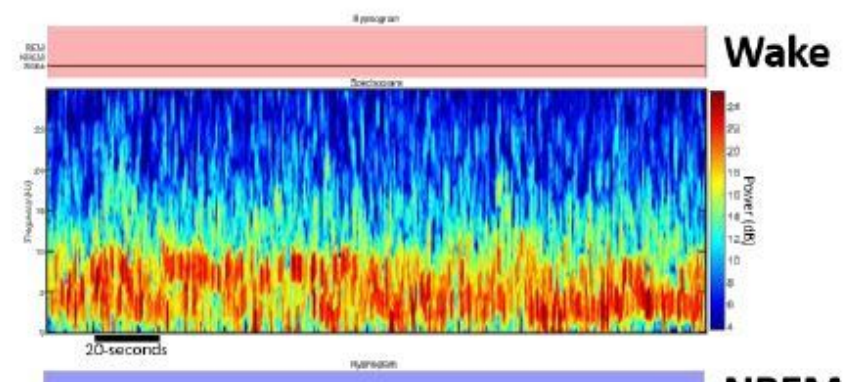

NREM

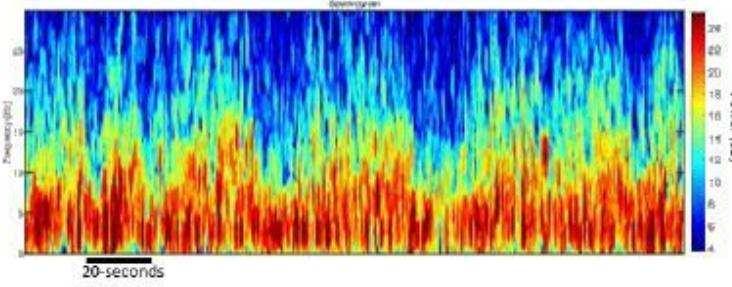

REM

\section{Figure 2}

The multitaper spectrogram reflects the vigilance status of control mice. (A) A representative multitaper spectrogram and a hypnogram of 24-hour EEG recording of a 6-month-old control mouse. In the hypnogram (top), pink shades indicate wakefulness, purple NREM sleep, and REM sleep green. The spectrogram (middle and bottom) is designed with the 9 DPSS tapers having window length $(\mathrm{N})$ of $2.5 \mathrm{~s}$ with the step of $0.05 \mathrm{~s}$ and frequency resolution $(\Delta \mathrm{f})$ of $4 \mathrm{~Hz}$ and bandwidth $(T W)=5$, and power is described in $\mathrm{dB}$ with provided color scale bars. (bottom) An enlarged one-minute segment of the spectrogram from zeitgeber time 8 to 9 . The detected TF-peaks were labeled with the black dotted line on the spectrogram. Rectangles with black dotted lines denote sigma TF-peaks. (B) Representative multitaper spectrograms of 200 second period spent in wakefulness (pink), NREM sleep (purple), and REM sleep (green). 


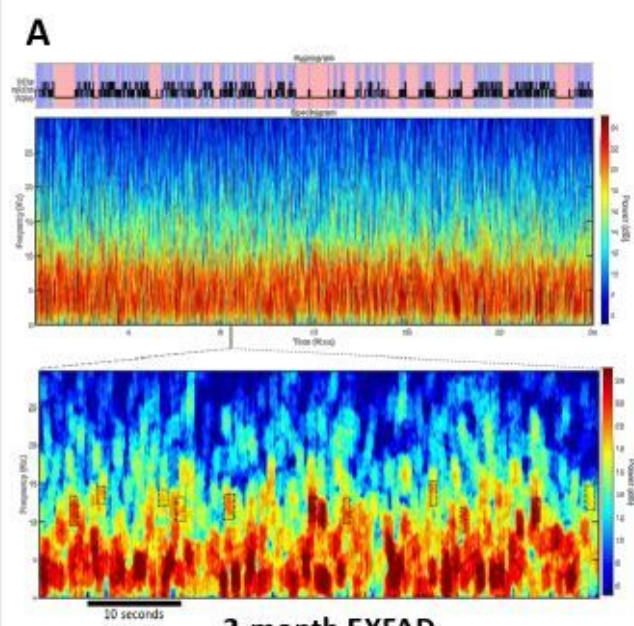

3-month 5XFAD
B
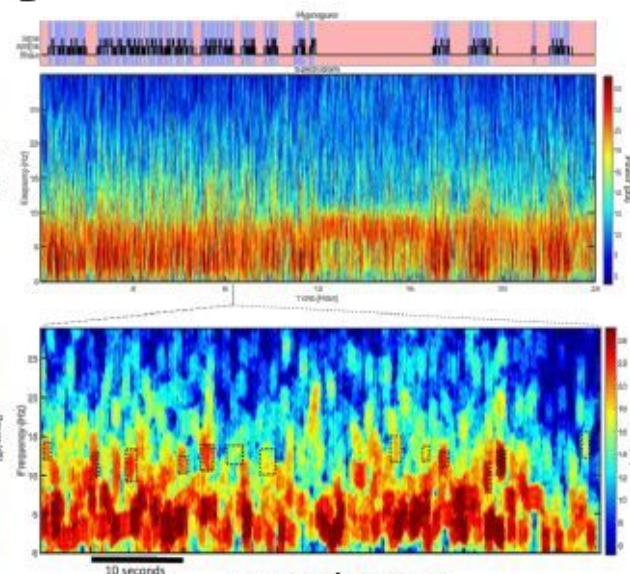

6-month 5XFAD
C
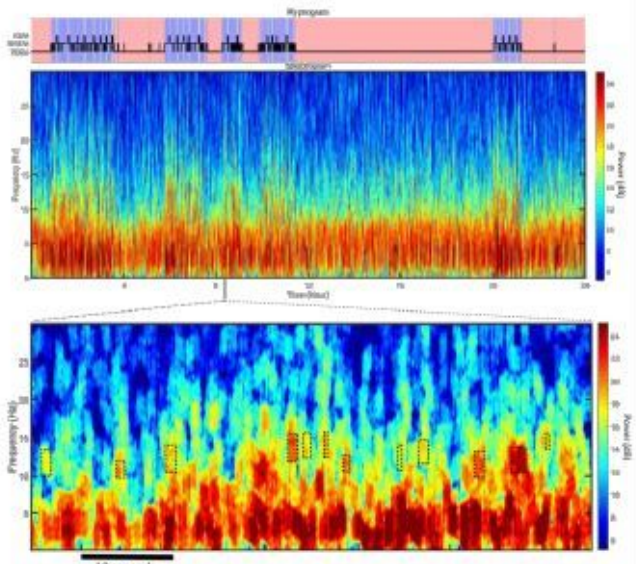

10-month 5XFAD

\section{Figure 3}

The longitudinal changes of EEG in 5XFAD mice by the multitaper spectrogram. (A) A representative multitaper spectrogram and a hypnogram of 24-hour EEG recording of a 3-month-old control mouse. In the hypnogram (top), pink shades indicate wakefulness, purple NREM sleep, and REM sleep green. The spectrogram (middle and bottom) is designed with the 9 DPSS tapers having window length $(N)$ of $2.5 \mathrm{~s}$ with the step of $0.05 \mathrm{~s}$ and frequency resolution $(\Delta \mathrm{f})$ of $4 \mathrm{~Hz}$ and bandwidth $(T W)=5$, and power is described in $\mathrm{dB}$ with provided color scale bars. (bottom) An enlarged one-minute segment of the spectrogram from zeitgeber time 8 to 9. (B and C) Figures with same formats of a representative 6- and 10-month-old 5XFAD mouse, respectively. 

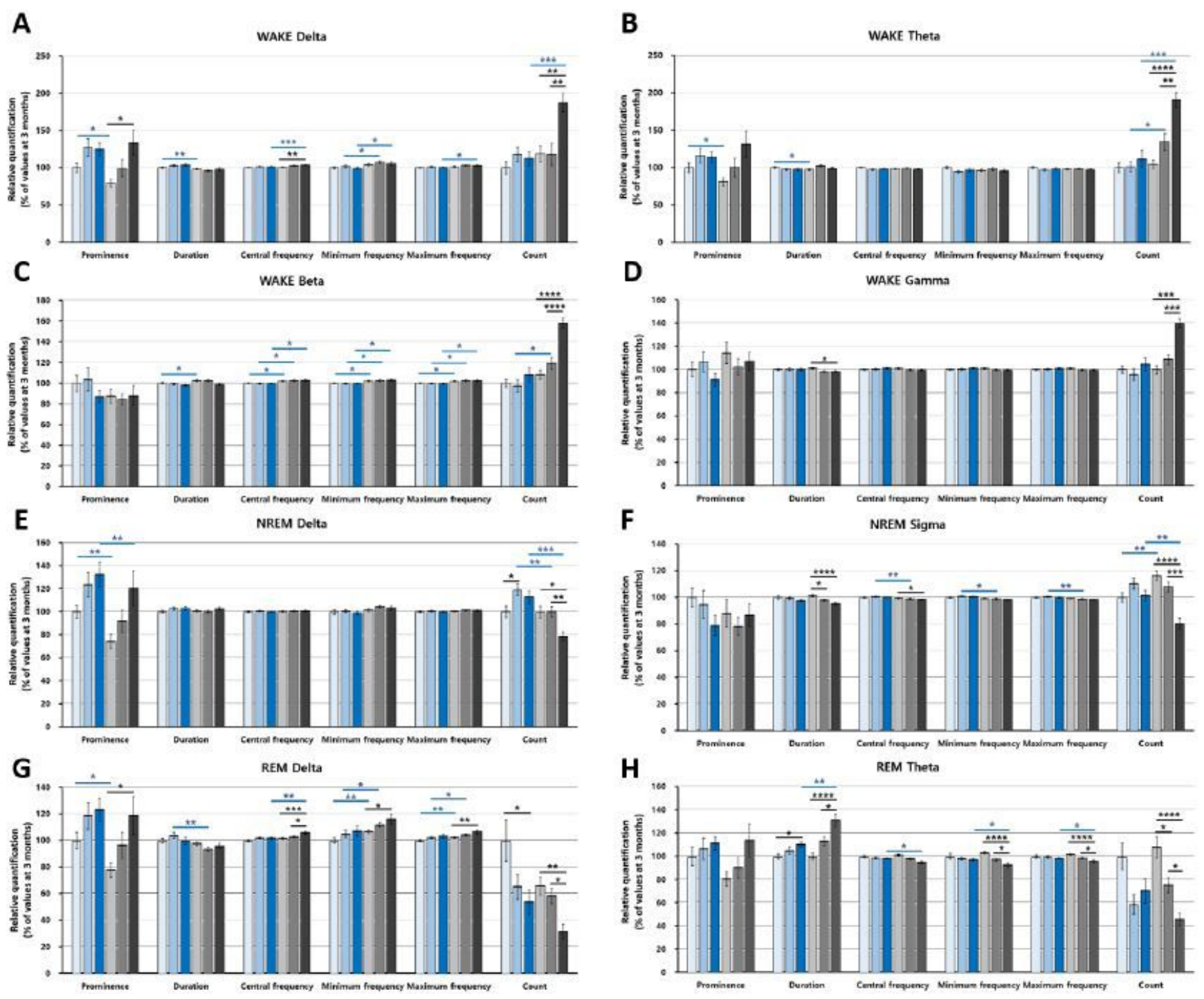

$\square 3$ Month Control $\square 6$ Month Control $\square 10$ Month Control

$\square 3$ Month 5XFAD $\square 6$ Month 5XFAD $\square 10$ Month 5XFAD

\section{Figure 4}

Morphological parameters of original and revised sigma TF peaks and sleep spindles. (A-C) Control mice. (D-F) 5XFAD mice. (A and D; B and E; C and F) Age groups of 3, 6, 10 months, respectively. (A-F) Histograms of morphological parameters for the sigma TF peaks, including log prominence (upper left), central frequency (upper right), duration (lower left) for each panel. Red lines indicate the histogram of the original sigma TF peak, blue sleep spindle, and green revised TF peak (duration $>0.5 \mathrm{~s}$ ). 


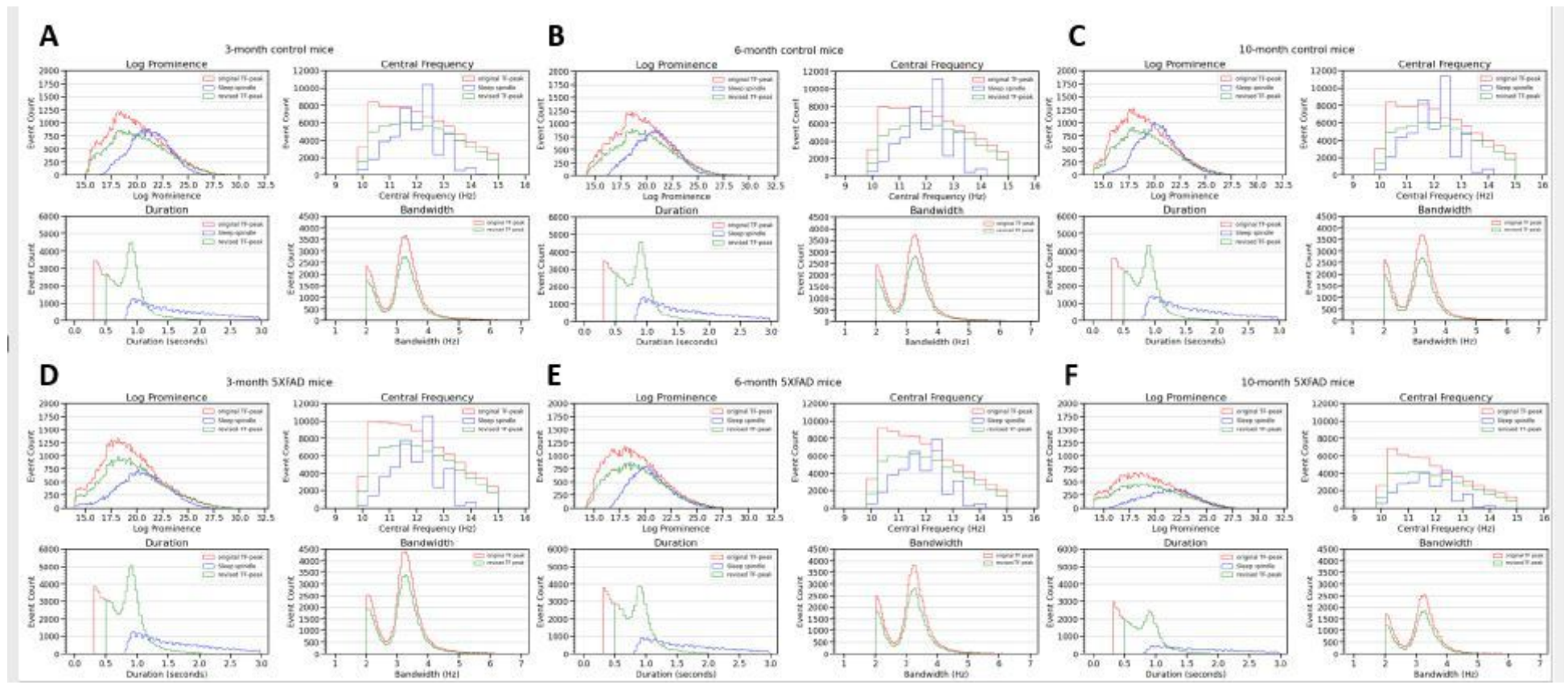

\section{Figure 5}

Morphological parameters of the bandwise TF peaks during wakefulness, NREM and REM sleep in control and 5XFAD mice. (A-D) Genotype and age group comparisons of morphological parameters, count, state time, and density for delta, theta, beta, and gamma frequency bands. Parameters were normalized to the value of 3-month-old control mice. ( $E$ and F) Same comparisons of delta and sigma TF peaks during NREM sleep. ( $G$ and $H$ ) Same comparisons of delta and theta TF peaks during NREM sleep. Blue horizontal lines indicate the comparison between genotypes; black horizontal lines between age

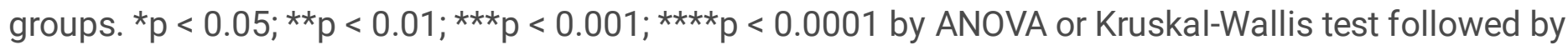
post hoc pairwise comparisons. 


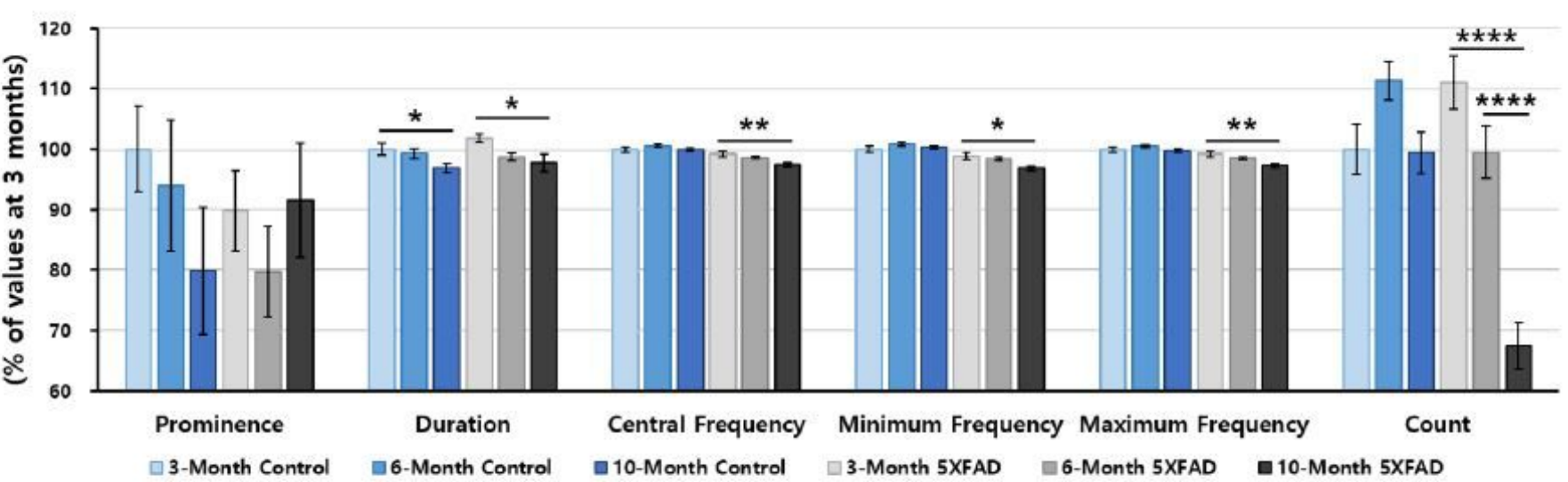

B

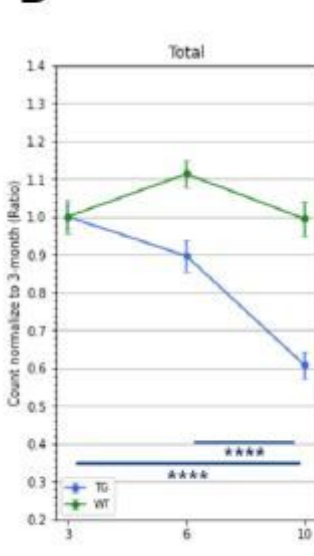

Normalized SXFAD TF-peak Counts fast / Light
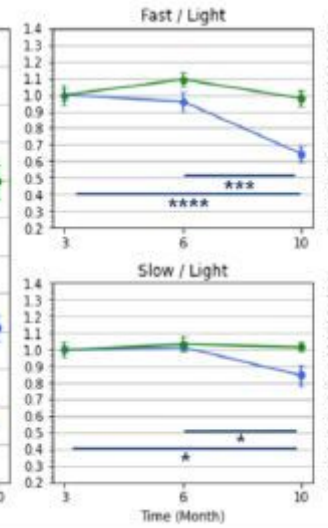

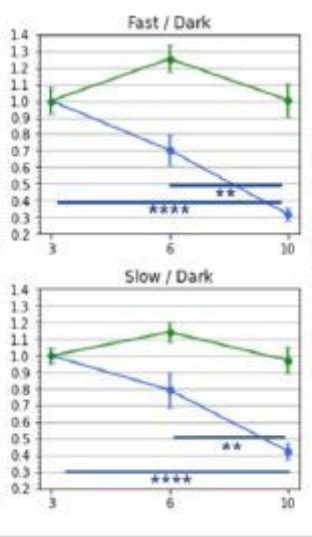

C

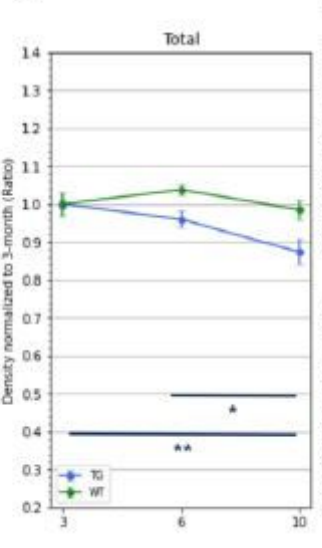

Nomalized 5XFAD TF-peak Density
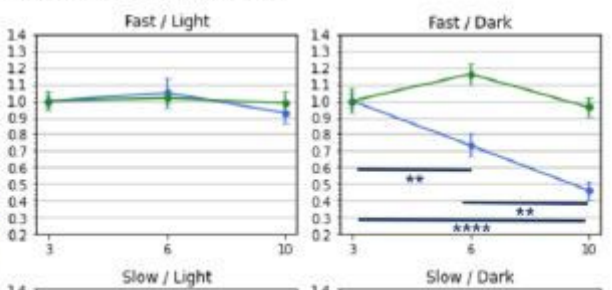

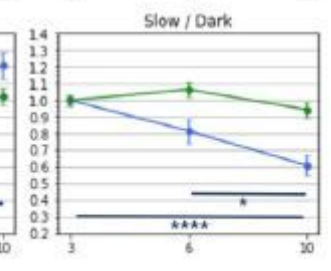

\section{Figure 6}

Parameters of TF peaks for sleep spindles and subgroup analysis. (A) Morphological parameters of sleep spindle frequency range $(9-17 \mathrm{~Hz})$. Parameters were normalized to the value of 3-month-old control mice. (B and C) The count and the density of TF peaks for fast $(9-12 \mathrm{~Hz})$ and slow $(12-17 \mathrm{~Hz})$ spindle frequency during light and dark periods. Green dots and lines indicate 5XFAD mice and blue dots and lines control mice. ${ }^{*} p<0.05 ;{ }^{* \star} p<0.01 ;{ }^{* \star *} \mathrm{p}<0.001 ;{ }^{* \star \star \star} \mathrm{p}<0.0001$ by ANOVA or Kruskal-Wallis test followed by post hoc pairwise comparisons. 